\title{
Abordagem icnológica como ferramenta didática para avaliação ecológica
}

\author{
AN ICHNOLOGICAL FRAMEWORK AS A DIDACTIC TOOL FOR ECOLOGICAL EVALUATION
}

\author{
Paulo R.F. Souto, Elivelton J. Neves \& David N. N. A. Silva \\ 1 - Departamento de Ciências Naturais, Universidade Federal do Estado do Rio de Janeiro, Av. Pasteur, 458, Rio de Janeiro, Brasil. \\ E-MAIL: PRFSOUTO15@GMAIL.COM, DAVIDNETININS@GMAll.COM
}

\begin{abstract}
The relevance of laboratory practices in the development of scientific attitudes and competences in schools has led to the implementation of different strategies and development of new didactic approaches. Ichnological experiments contribute to analyze diverse sets of interrelated scientific data, such as: morphological structure of the organism, movement patterns, quality of produced pathways and substrate composition. The analysis of the impressions produced allows us to identify how different environmental conditions, such as humidity and soil compaction, can affect the preservation and structure of traces left by different organisms in the environment. The proposal seeks to increase practical activity by carrying out an experiment that uses four different arthropods, with different body shapes in the adult stage: centipede (Scolopendrasubspinipes), beetle (Hylobiusabietis), wolf spider (Lycosa sp.) and millipede (Jullus sp.), as well as highlight the relationship between biological and environmental aspects, making it possible to stimulate teaching and learning, emphasizing the relevance of the soil in the structuring of the biosphere.
\end{abstract}

Resumo: A relevância das práticas de laboratório no desenvolvimento de atitudes e competências científicas nas escolas levou à implementação de diferentes estratégias e ao desenvolvimento de novas abordagens didáticas. Experimentos icnológicos possibilitam analisar conjuntos diversificados de dados científicos inter-relacionados, tais como: estrutura morfológica do organismo, padrões de movimento, qualidade das vias produzidas e composição do substrato. A análise das impressões produzidas permite identificar de que maneira diferentes condições ambientais, como umidade e a compactação do solo, podem afetar a preservação e estrutura dos traços deixados por diferentes organismos no ambiente. A proposta busca incrementara atividade prática pela realização de experimento que utiliza quatro artrópodes diferentes, com diferentes formas corporais no estágio adulto: centopéia (Scolopendra subspinipes), besouro (Hylobius abietis), aranha (Lycosa sp.) e lacraia (Jullus sp.), bem como evidenciar a relação entre aspectos biológicos e ambientais, possibilitando estimular 0 ensino-aprendizagem, enfatizando a relevância do solo na estruturação da biosfera.
Citation/Citação: Souto, P. R.F., Neves, E. J., \& Silva, D. N. N. A. (2020). Abordagem icnológica como ferramenta didática para avaliação ecológica. Terræ Didatica, 16, 1-6, e020026. doi: 10.20396/ td.v16i0.8658922

Keywords: Arthropods. Ethology. Ichnology. Traces.

Palavras-chave: Artrópodos. Etologia. Icnologia. Pistas.

Manuscript/Manuscrito:

Received/Recebido: 28/03/2020

Revised/Corrigido: 04/05/2020

Accepted/Aceito: 10/06/2020

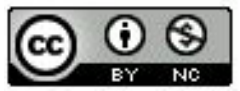

\section{Introdução}

A icnologia é uma ciência dedicada ao estudo das estruturas biogênicas produzidas a partir da atividade vital dos organismos que refletem a interação entre os organismos e o substrato. As alterações produzidas no sedimento, classificadas como bioturbações, são caracterizadas por: pistas, galerias, ninhos, câmaras e bolotas fecais, e afetam significativamente as propriedades dos solos, bem como a disponibilidade de recursos inorgânicos existentes. A tendência dos organismos de se distribuir nos ecossistemas conforme suas necessidades fisiológicas e ecológicas permi- te, inclusive, diferenciar os padrões de vida em relação ao substrato em epibiontes e endobiontes (Hasiotis, 2003). Diferentemente do ambiente marinho, os organismos de ambientes terrestres possuem diferentes necessidades de água e permeabilidade no substrato, concentração salina e iônica no sedimento aquoso ou em superfície e, consequentemente os animais, possuem diferentes estratégias e motivação, nesse sentido os estudos de neoicnologia também atuam como uma ferramenta útil na investigação dos processos que resultam em variações morfológicas dos traços fósseis, como variações no comportamento

\begin{tabular}{c|c|c|c|c|c}
\hline (C) Terrae Didat. & Campinas, SP & v.16 & $1-6$ & $\mathrm{e} 020026$ & 2020 \\
\hline
\end{tabular}


e nas condições do substrato no momento da sua produção, além de fatores de preservação, como falhas na trilha e resistência(Martin \& Rindsberg, 2006). Para compreender a dinâmica de uma bioturbação é essencial considerar fatores entre os quais: a morfologia, a abundância e diversidade da macrofauna existente, os quais estão subordinados a vários aspectos, como tipo do solo, temperatura, umidade, cobertura vegetal, ação humana, sedimentação, topográfica e climática, (Wolters, 2000; Anderson, 2009).

A análise das estruturas geradas no substrato permite estabelecer associações entre o padrão de movimento, a morfologia do produtor e possíveis estruturas biogênicas resultantes no intuito de fornecer referenciais ao estudo das impressões produzidas pelos diferentes animais presentes em determinada área (Donovan, 1994). Entretanto, a validade das interpretações torna-se mais precisa quando são fundamentadas em evidências experimentais realizadas em laboratório; para tanto, é essencial o desenvolvimento de metodologias que sirvam de parâmetros nas pesquisas de campo e que possam ter aplicabilidade didática. As experiências envolvendo artrópodes receberam pouca atenção e estão concentradas nas escavações de tocas (Chamberlain, 1975, Elders, 1975, Frey \& Pemberton, 1991). Os estudos de pistas de artrópodes atuais foram, em geral, no sentido de auxiliar na interpretação descritiva dos registros fossilizados, relacionados a aracnídeos (Brand \& Kramer, 1996, Alf, 1968, Sadler, 1993), crustáceos (Margerard, 2000, Walker et al., 2003 Gaillard et al. 2005) e miriápodes (Johnson et al. 1994). As pistas de hexápodes atuais foram registradas, no propósito de dedução das técnicas de locomoção (Manton, 1972), ou de hábito oportunista (Metz, 1987).

No Brasil, observa-se uma preocupação crescente com a qualidade do ensino básico. Diversas propostas têm sido desenvolvidas a fim de ampliar a divulgação e popularizar os conhecimentos científicos, a fim de expandir a utilização das informações obtidas através de pesquisas nas universidades pela sociedade em geral. O objetivo deste estudo é aproximar e despertar o interesse dos professores e alunos principalmente em geociências integrando os conhecimentos científicos, neste caso da icnologia, por meio de um experimento acessível e que possa ser inserido no cotidiano escolar, a fim de incentivar a realização de formas alternativas de ensino e divulgação das Geociências.

\section{Material e Métodos}

O procedimento experimental desenvolvido no laboratório de Interações Biológicas e Ambientais, contou com a participação de alunos monitores do curso de graduação do Instituto de Biociências da UNIRIO, e seguiu a metodologia de sistema transacional subaquoso-subaéreo proposto em Davis et al. (2007), sendo utilizado quatro espécies de artrópodes terrestres atuais, com formas corporais diferenciadas e na fase adulta: besouro (Hylobius abietis), centopéia (Jullus sp.), lacraia (Scolopendra subspinipes) e caranguejeira (Loxosceles sp.). Os artrópodes foram pesados em balança digital (Shimadsu) e medidos com paquímetro digital (Mitutoyo) conforme apresentado na tabela 1. A terminologia utilizada na descrição das marcas e pistas dos artrópodes seguiram a terminologia proposta por Minter (2007). Os artrópodes escolhidos podem ser facilmente encontrados em jardins e matas próximas de áreas urbanas o que permite sua reposição, entretanto, ao longo dos experimentos os animais fiquem alojados em terrários separadamente (Carvalho \&Cavalgo, 2002). Os substratos utilizados foram coletados em diferentes ambientes e consistiram de: lama (tamanho: $0,01 \mathrm{~mm}$ ), argila (tamanho: $0,0035 \mathrm{~mm}$ ) e areia fina (tamanho: $0,22 \mathrm{~mm}$ ). Para remoção de impurezas, os sedimentos foram passados em peneiras com malha $1 \mathrm{~cm}$, e acomodados em bandejas plástica de polietileno com 56×35×12 $\mathrm{cm}$, e com caixas internas de madeira impermeável para impressão das pistas com 29x21 $4.5 \mathrm{~cm}$, e a altura do sedimento no interior das bandejas correspondendo a $9 \mathrm{~cm}$. Os artrópodes utilizados apresentaram peso de: $0,26 \mathrm{~g}$ no aracnídeo, $0,92 \mathrm{~g}$ no coleóptero, 4,29g no diplópode e $1,22 \mathrm{~g}$ no quilópode. Os experimentos consistiram em induzir os animais ao deslocamento sobre o substrato por meio de estímulos mecânicos com toque de pinça ou pelo direcionamento de fonte luminosa de uma lanterna, em ambiente escuro, nas bandejas contendo os substratos. As impressões produzidas pelos artrópodes foram avaliadas na condição seca

Tabela 1. Especificações do peso e do comprimento dos artrópodes utilizados no estudo

\begin{tabular}{l|c|c}
\hline \multicolumn{1}{c|}{ Artrópodes } & $\begin{array}{c}\text { comprimento } \\
(\mathrm{mm})\end{array}$ & $\begin{array}{c}\text { peso } \\
(\mathrm{g})\end{array}$ \\
\hline Hylobius abietis & 32.3 & 6.0 \\
\hline Jullus sp. & 82.5 & 4.3 \\
\hline Scolopendra subspinipes & 65.4 & 1.2 \\
\hline Lycosa sp. & 12.2 & 1.0 \\
\hline
\end{tabular}


$0 \mathrm{ml} / \mathrm{cm}^{2}$ e de saturação de água de $1 \mathrm{ml} / \mathrm{cm}^{2}$ e $2 \mathrm{ml} /$ $\mathrm{cm}^{2}$ a fim de proporcionar diferentes condições de plasticidade. Após o deslocamento os animais eram reconduzidos aos terrários e os rastros fotografados.

\section{Discussão e Resultados}

A morfologia dos rastros produzidos em superfície de condição plana e nitidez foram caracterizadas: no lacraia (Scolopendrasubspinipes), rastros paralelos e simétricos com várias pegadas circulares, com muita sobreposição, e três rastros centrais contínuos, sendo que apenas o do meio é formado pelo corpo do animal, e os outros dois também são formados pelas pernas, na aranha ( $L$ cosa sp.), rastro com três a quatro pares de pegadas, sendo o quarto par sempre visível, com a forma alongada, e os outros pares com formato circular. Há ocasionalmente uma sobreposição das pegadas dos 18 pares e, portanto, nem sempre ambos são visíveis. A trilha possui simetria alternada e é bem definida. (Fig. 1), na centopéia (Jullus sp.), deixou um rastro bem nítido, com pegada de simetria oposta, composto por uma sequência de impressões de formato alongado ou elipsóide, muito próximas e com muita sobreposição, e um rastro central contínuo formado pelo arraste do abdômen do animal (Fig. 1), e no besouro (Hylobius abietis), deixou impressões acentuadas, devido às pernas pontiagudas que penetram profundamente a areia a cada passo, sendo possível ver pegadas de dois a três pares de pernas, com formato alongado, simetria oposta e pouca sobreposição, e uma impressão central continua, formada pelo arraste do abdômen do animal, conforme consta das Figuras 2, 3, 4, 5. Os percentuais encontrados de rastros visíveis produzidos nas três condições de substratos foram: para o diplópode $13,8 \%$ e o coleóptero $8,3 \%$, seguidos em menor percentual pelo quilópode $5 \%$ e a aranha $2,5 \%$.

No experimento verifica-se que os fatores que contribuem significativamente na produção e visibilidade dos rastros estão relacionados ao peso do animal e à umidade dos substratos, sendo avaliado quantitativamente nos substratos secos de $40 \%$ dos rastros identificáveis, nos substratos com saturação parcial de $1 \mathrm{ml} / \mathrm{cm}^{2}$ de $13 \%$ das marcas razoavelmente visíveis, e nos substratos muito saturados de $2 \mathrm{ml} / \mathrm{cm}^{2}$ de apenas $8 \%$ das marcas produzidas. Quanto à morfologia corporal dos artrópodes utilizados, a maior influência foi verificada no substrato seco com rastros muito distintos, sendo possível constatar ainda,nessa condição, que o peso e a superfície plantar contribuem para maior precisão da produção do rastro, como no caso o coleóptero e o diplópode que apresentam peso acima de uma grama.
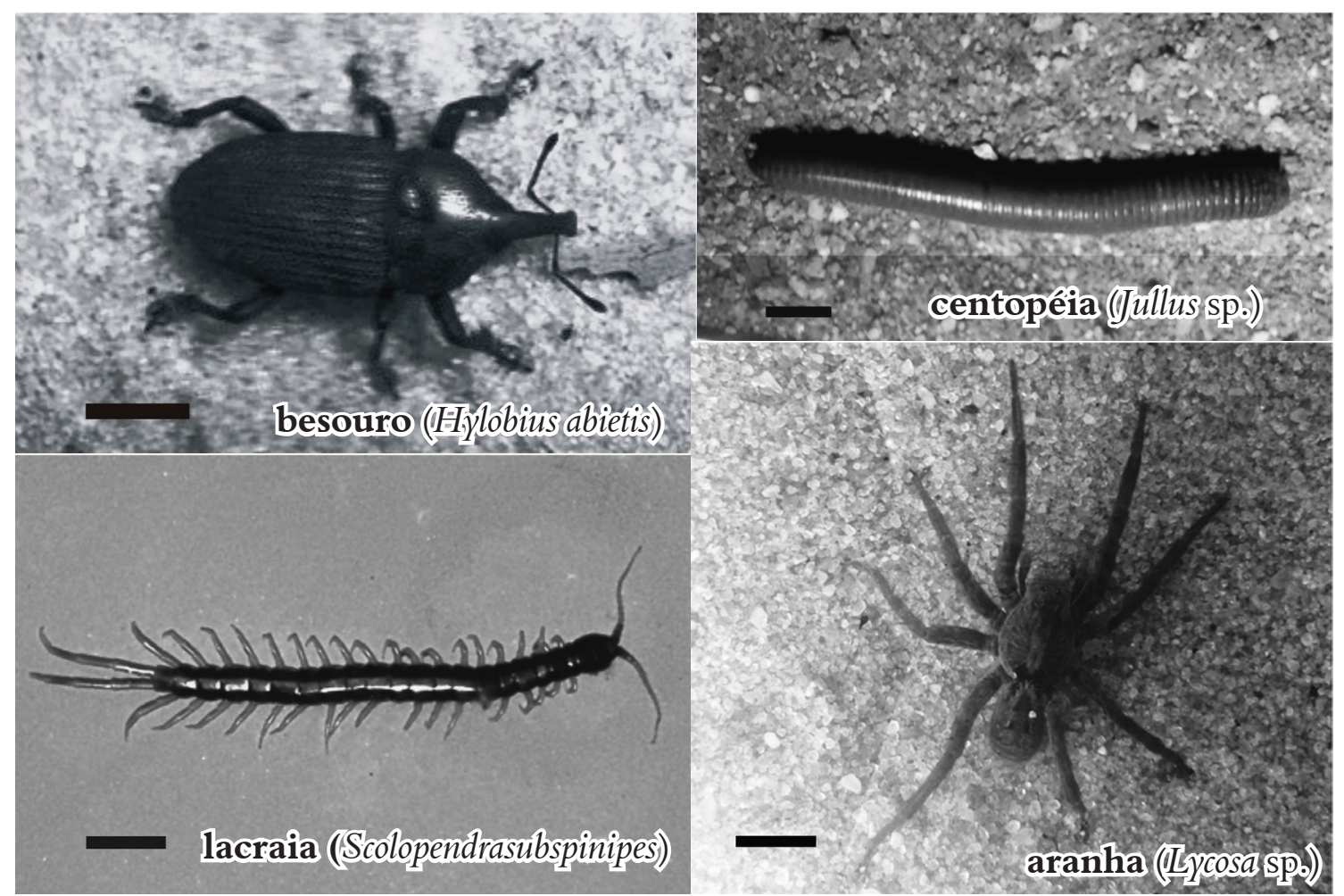

Figura 1. Artrópodes utilizados no experimento. Escala: $10 \mathrm{~mm}$ \begin{tabular}{l|l} 
(c) Terrae Didat. & Campinas, SP
\end{tabular}

v.16

$1-6$

e020026

2020 


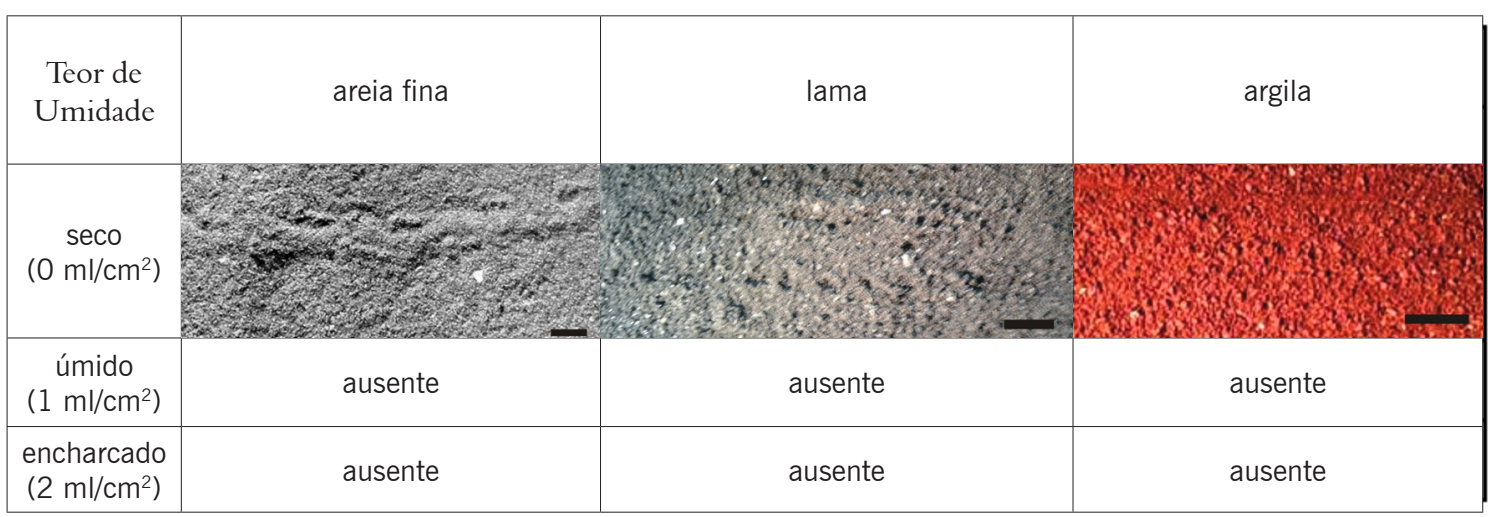

Figura 2. Diagrama da presença de pistas produzidas por lacraia (Scolopendra subspinipes) em relação ao teor de umidade no substrato. 0 animal se deslocou da esquerda para a direita em todos os casos. Escala: $10 \mathrm{~mm}$

Entretanto, em substratos que sofreram aumento da umidade, as impressões não permitiram uma diferenciação quanto ao tipo de produtor devido à falta de nitidez das impressões. Todos os deslocamentos realizados no substrato com argila úmida ou encharcados, não produziram marcas continuas ou visíveis, provavelmente devido à menor plasticidade da superfície e redução do atrito no deslocamento dos artrópodes utilizados. Desse modo é possível concluir que nos três substratos saturados de umidade a mecânica natural do movimento foi visivelmente alterada e em quase todos os rastros inadequados a produção de marcas, com alguma exceção para o besouro e a centopéia que produziram alguns rastros indiferenciados.

Os resultados também revelam que os rastros deixados por estes e outros grupos de animais podem mudar drasticamente em consequência de fatores como tipo de substrato, teor de umidade, irregularidade do terreno e nível de estresse do animal (letargia ou fuga). Assim, em situações diferentes, alguns produziram rastros semelhantes entre si e semelhantes aos dos outros artrópodes utilizados. Os experimentos indicam que em determinadas condições é muito difícil atribuir um rastro a um tipo particular de artrópode, pois quando o animal faz mais esforço as pegadas ficam sobrepostas, como observado no substrato mais úmido, ou quando o peso não é significativo, os padrões ficam muito semelhantes até mesmo entre classes distintas, como verificado nesse estudo nos rastros de coleópteros e diplópodes na lama úmida (figura 4 e5).

A proposta do experimento permite concluir em primeiro enfoque, que o monitoramento iconológico realizado em laboratório pode ser empregado como uma ferramenta que contribui na construção do saber e na percepção do aluno de forma pedagógica por propiciar no entendimento a respeito da mecânica envolvida no movimento de diferentes animais e na noção dos vários aspectos sedimentares relacionados a estrutura dos substratos, e em segundo, na perspectiva que também os icnitos preservados em sítios fossilíferos, auxiliam no entendimento sobre a distribuição e composição das comunidades extintas. Desse modo, esse

\begin{tabular}{|c|c|c|c|}
\hline $\begin{array}{c}\text { Teor de } \\
\text { Umidade }\end{array}$ & areia fina & lama & argila \\
\hline $\begin{array}{c}\text { seco } \\
\left(0 \mathrm{ml} / \mathrm{cm}^{2}\right)\end{array}$ & & ausente & ausente \\
\hline $\begin{array}{c}\text { úmido } \\
\left(1 \mathrm{ml} / \mathrm{cm}^{2}\right)\end{array}$ & ausente & ausente & ausente \\
\hline $\begin{array}{c}\text { encharcado } \\
\left(2 \mathrm{ml} / \mathrm{cm}^{2}\right)\end{array}$ & ausente & ausente & ausente \\
\hline
\end{tabular}

Figura 3. Diagrama da presença de pistas produzidas por caranguejeira (Loxosceles sp.) em relação ao teor de umidade no substrato. $O$ animal se deslocou da esquerda para a direita em todos os casos. Escala: $10 \mathrm{~mm}$

\begin{tabular}{c|c|c|c|c|c|}
\hline C Terrae Didat. & Campinas, SP & v.16 & $1-6$ & $\mathrm{e} 020026$ & 2020 \\
\hline
\end{tabular}




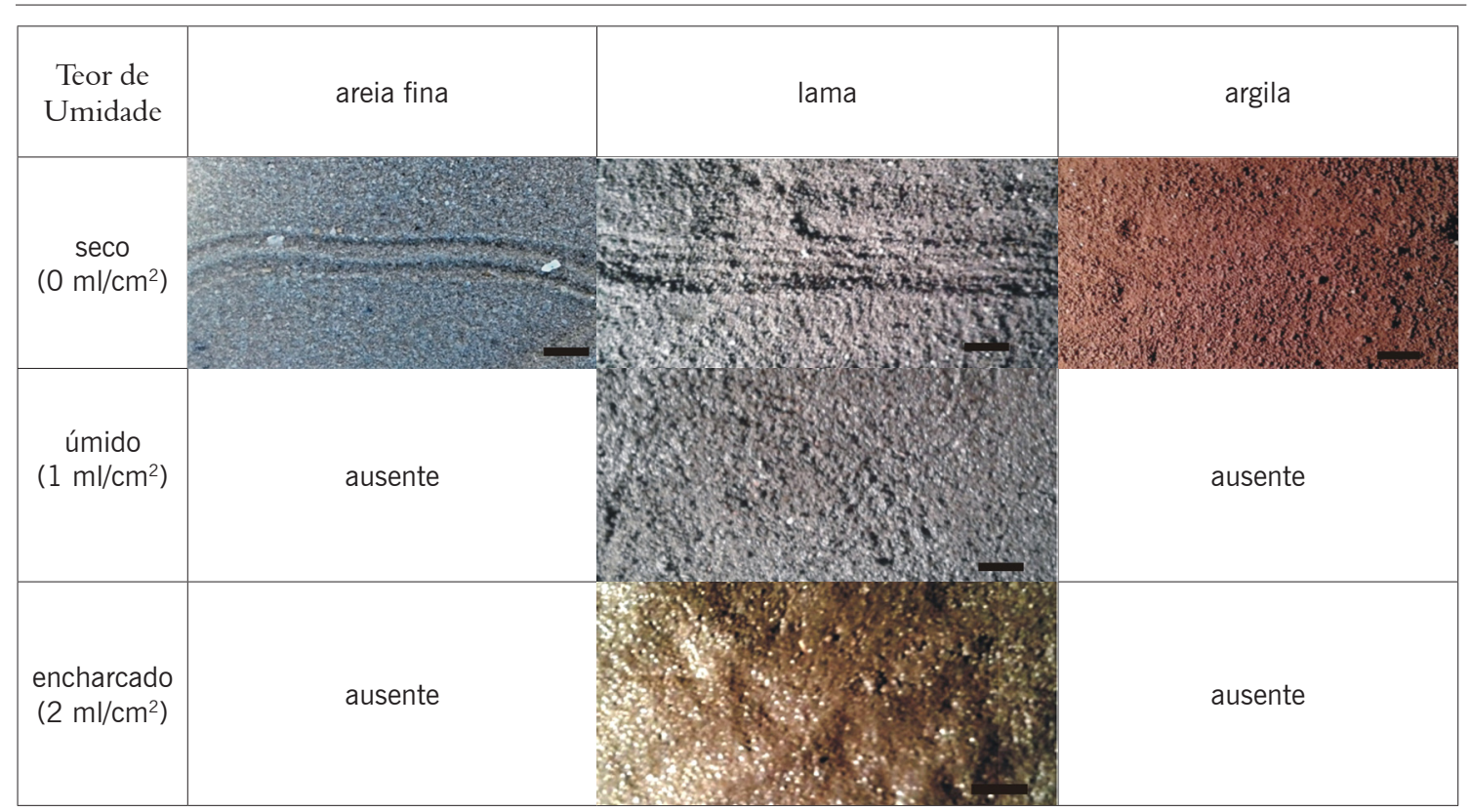

Figura 4. Diagrama da presença de pistas produzidas por centopéia (Jullus sp.) em relação ao teor de umidade no substrato. 0 animal se deslocou da esquerda para a direita em todos os casos. Escala: $10 \mathrm{~mm}$

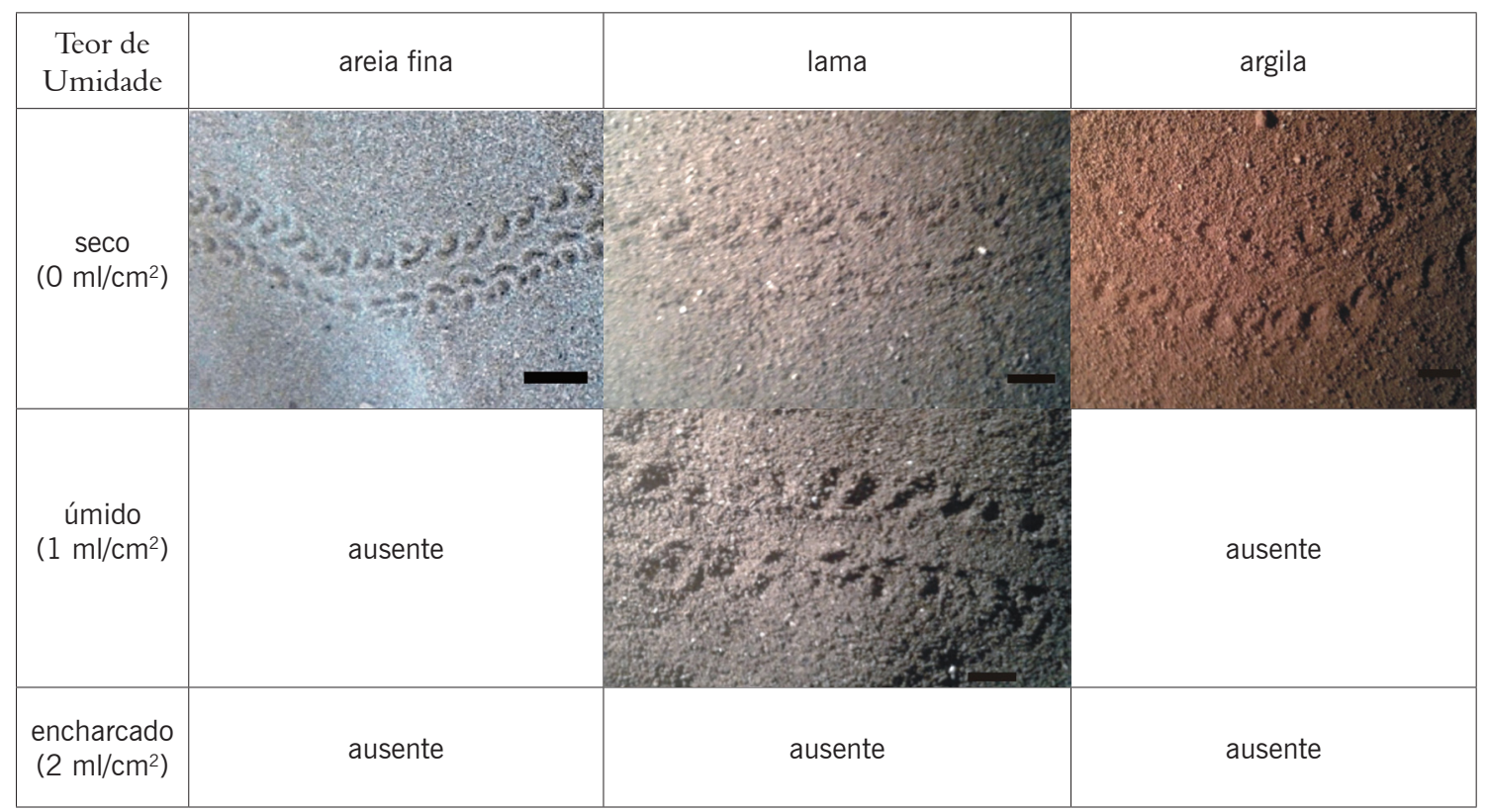

Figura 5. Diagrama da presença de pistas produzidas por besouro (Hylobius abietis) em relação ao teor de umidade no substrato.

$\mathrm{O}$ animal se deslocou da esquerda para a direita em todos os casos. Escala: $10 \mathrm{~mm}$

estudo abrange níveis de ensino variados e contribui indiretamente na formação da capacidade de investigação biológica e no entendimento da adaptação dos organismos ao meio ambiente.

\section{Agradecimentos}

Os autores agradecem ao Programa de Incentivo Acadêmico do Departamento de Ciências Naturais, pelo auxílio financeiro fornecido; ao apoio do prof. Dr. Elidiomar Ribeiro da Silva do Laboratório de Entomologia Urbana e Cultural da Universidade Federal do Estado do Rio de Janeiro e às sugestões dos revisores de Terre Didatica.

\section{Referências}

Alf, R. M. (1968). A spider trackway from the Coconino Formation, Seligman, Arizona. Bulletin of the Southern California Academy of Sciences, 67(2), 125-128. URL: https://scholar.oxy.edu/scas/vol67/iss2/9/. 
Anderson, J. M. (2009). Why should we care about soil fauna? Pesquisa de Agropecuária Brasileira, 44(8), 835842. doi: 10.1590/S0100-204X2009000800006.

Brand, L. R. \& Kramer, J.(1996). Underprints of vertebrate and invertebrate trackways in the Permian Coconino sandstone in Arizona. Ichnos, 4(3), 225230. doi: 10.1080/10420949609380129.

Carvalho, M. C. N. \& Carvalho, R. I. N. (2002). Terrários: Ciência e Arte. Curitiba, Ed. UFPR. 67p.

Chamberlain, C. K. (1975). Recent lebensspuren in nonmarine aquatic environments. In: Frey, R. W. (ed.). (1975). The Study of Trace Fossils: A Synthesis of Principles, Problems and Procedures in Ichnology. New York, Springer-Verlag. p. 431-58.

Davis, R. B., Minter, N. J., \& Braddy, S. J. (2007). The neoichnology of terrestrial arthropods. Palaeogeography, Palaeoclimatology, Palaeoecology, 255, 284-307. doi: 10.1016/j.palaeo.2007.07.013

Donovan, S. K. (1994). The Palaeobiology of Trace Fossils. New York, Wiley-Blackwell. 604p.

Elders, C. A. (1975). Experimental approaches in neoichnology. In: Frey, R.W. (ed.) (1975). The Study of Trace Fossils: A Synthesis of Principles, Problems and Procedures in Ichnology. New York, Springer-Verlag. p. 513-36.

Frey, R. W., \& Pemberton, S. G. (1991). The ichnogenus Schaubcylindrichnus: morphological, temporal, and environmental significance. Geological Magazine, 128(6), 595-602. doi: 10.1017/ s0016756800019713.

Gaillard, C., Hantzpergue, P., Vannier, J., Margerard, A. L., \& Mazin, J. M. (2005). Isopod trackways from the CrayssacLagerstätte, Upper Jurassic, France. Palaeontology, 48, 947-962. doi: 10.1111/j.1475$-4983.2005 .00502 . x$.

Hasiotis, S.T. (2003). Complex ichnofossils of solitary and social soil organisms: understanding their evolution and roles in terrestrial paleoecosystems. Palaeogeography, Palaeoclimatology, Palaeoecology, 192, 259-320. doi: 10.1016/s0031-0182(02)00689-2.

Johnson, E. W., Briggs, D. E. G., Suthren, R .J., Wright, J., \& Tunnicliff, S. P. (1994). Non-marine arthro- pod traces from the subaerial Ordovician Borrowdale Volcanic Group, English Lake District. Geological Magazine, 131, 395-406. doi: 10.1017/ s0016756800011146.

Manton, S. M. (1972). The evolution of arthropodan locomotory mechanisms: Part 10. Locomotory habits, morphology and evolution of hexapod classes. Zoological Journal of the Linnean Society, 51, 257 375. doi: 10.1111/j.1096-3642.1973.tb00790.x.

Margerard, A. L. (2000). L'isopode Archaeoniscus (Crustacea) du Jurassique supérieur O-européen (France, Grande-Bretagne): anatomie, ichnologie, modélisationbioméchanique. Lyon, Université de Lyon, France. 245p. (MSc dissertation).

Martin, A. J., \& Rindsberg, A. K. (2006). Arthropod tracemakers of Nereites? Neoichological observations of juvenile limulids and their paleoichnological applications. In: Miller, W. (ed.). (2006). Trace Fossils: Concepts, Problems, Prospects. Amsterdam, Elsevier. p. 478-491.

Metz, R. (1987). Insect traces from nonmarine ephemeral puddles. Boreas, 16, 189-195. doi: 10.1111/ j.1502-3885.1987.tb00770.x.

Minter, N. J., Krainer, K., Lucas, S. G., Braddy, S. J.\& Hunt, A. P. (2007). Palaeoecology of an Early Permian playa lake trace fossil assemblage from Castle Peak, Texas, USA. Palaeogeography, Palaeoclimatology, Palaeoecology, 246, 390-423. doi: 10.1016/j.palaeo.2006.10.009.

Sadler, C. J. (1993). Arthropod trace fossils from the Permian DeChelly Sandstone, northeastern Arizona. Journal of Paleontology, 67, 240-249. doi: 10.1017/s0022336000032169.

Walker, S .E., Holland, S .M. \& Gardiner, L. (2003). Coenobichnus currani (new ichnogenus and ichnospecies): fossil trackway of a land hermit crab, Early Holocene, San Salvador, Bahamas. Journal of Paleontology, 77, 576582. doi: 10.1666/0022-3360(2003)077<0576:ccniai $>2.0 . \mathrm{co} ; 2$.

Wolters, V. (2000). Invertebrate control of soil organic matter stability. Biology and Fertility of Soils, 31, 1-19. doi: 10.1007/s003740050618. 